\title{
Time-Dependent Clinical Results of Rotating-Platform Total Knee Arthroplasty According to Mechanical Axis Deviation
}

\author{
Han-Jun Lee, MD, Ho-Joong Jung, MD, Young-Bok Jung, MD, Young-Bong Ko, MD, Min-Ku Song, MD, and \\ Seong-Hwan Kim, MD \\ Department of Orthopedic Surgery, Chung-Ang University Hospital, Seoul, Korea
}

\begin{abstract}
Purpose: We hypothesized that the low contact stress (LCS) posterior stabilization system in knees with $\leq 3^{\circ}$ deviation of coronal alignment would provide more favorable clinical outcomes and survival rate over the course of time.

Materials and Methods: A retrospective study was performed on 253 consecutive cases of primary total knee arthroplasty (TKA). Patients were classified according to the degree of deviation of coronal alignment on the initial postoperative radiograph as Group 1 ( $\leq 3^{\circ}$ deviation) and Group 2 ( $>3^{\circ}$ deviation). The clinical assessments were performed using the Knee Society score and Hospital for Special Surgery systems and Western Ontario and McMaster Universities index.

Results: The survival rate was $97.4 \%$ in Group 1 and $96.8 \%$ in Group 2. No statistically significant intergroup difference was observed in the clinical scores before surgery and since 1 year after surgery $(\mathrm{p}>0.05)$. However, a significant intergroup difference was noted between 6 months to 1 year after surgery $(\mathrm{p}<0.001)$. Less than $2 \mathrm{~mm}$ radiolucent lines were found more frequently in Group 2 . Time-dependent improvement was noted within one year after TKA in both groups.

Conclusions: Most of the expected improvements were achieved at 6 months after surgery in Group 1 and at 1 year after surgery in Group 2. The present study suggests that the LCS system yields time-dependent improvement regardless of coronal alignment deviation.
\end{abstract}

Keywords: Knee, Arthroplasty, Treatment outcome

\section{Introduction}

Total knee arthroplasty (TKA) using fixed-bearing implants has produced good long-term survival rates of up to $97 \%$ at 10 years ${ }^{1,2)}$. However, there have been problems of premature polyethylene wear and aseptic loosening with some fixed-bearing implants. To address this issue, mobile-bearing TKA was developed

Received December 9, 2013; Revised (1st) February 26, 2014;

(2nd) May 6, 2014; Accepted May 15, 2014

Correspondence to: Seong-Hwan Kim, MD

Department of Orthopedic Surgery, Chung-Ang University Hospital, 102 Heukseok-ro, Dongjak-gu, Seoul 156-755, Korea

Tel: +82-2-6299-1577, Fax: +82-2-822-1710

E-mail:ksh170177@nate.com

This is an Open Access article distributed under the terms of the Creative Commons Attribution Non-Commercial License (http://creativecommons.org/licenses/by-nc/3.0/) which permits unrestricted non-commercial use, distribution, and reproduction in any medium, provided the original work is properly cited. and has been performed worldwide for over 20 years.

The low contact stress mobile-bearing knee prosthesis (LCS system; DePuy Orthopaedics Inc., Warsaw, IN, USA) provides theoretical advantages over fixed-bearing prostheses. The mobile polyethylene insert, in the form of either twin meniscal bearings or a single rotating platform, permits increased congruity, ensures reduced or even no shear stress at the bone-implant interface, and is anticipated to reduce the prosthesis loosening rate. Midand long-term studies of the LCS system showed as satisfactory results as fixed-bearing $\mathrm{TKA}^{3-5)}$.

Correct alignment of the components including soft tissue balancing is crucial for successful knee arthroplasty ${ }^{6}$. Various ranges of acceptable limb alignment have been reported, but $\pm 3^{\circ}$ varus/ valgus mechanical axis has been associated with higher success rates $^{7,8)}$. The postoperative limb alignment can be over $\pm 3^{\circ}$ varus/ valgus in up to $30 \%$ of the cases after conventional TKAs ${ }^{9}$.

Information on the implant survival rate and ideal postoperative alignment are important to surgeons and patients for ap- 


\section{Lee et al. Time Dependent Improvement in TKA with LCS System}

propriate treatment planning; however, from the patient's point of view, it is equally important to know how well the knee will function after a TKA. Recent studies focusing on patient-relevant functional outcome of TKA have provided some information on the postoperative recovery: several factors including preoperative function, gender, and, especially, recovery time influenced clinical outcomes regardless of the alignment ${ }^{10-12}$.

Moreover, the recent concept of constitutional varus alignment in normal people has made surgeons to reconsider whether restoration of neutral coronal alignment is desirable for all patients. In the study by Bellemans et al. ${ }^{13)}$, a large fraction of the normal population (32\% of men and $17 \%$ of women) had constitutional varus alignment: therefore, they suggested the postoperative alignment target should be individualized according to the patient's preoperative knee alignment, which does not necessarily lie within $0^{\circ} \pm 3^{\circ}$ in reference to the neutral mechanical axis. Likewise, in a recent clinical study on TKA, Parratte et al. ${ }^{14)}$ demonstrated there was no difference in the 15-year implant survival rate between the group with a mechanical axis of $\pm 3^{\circ}$ and the outlier group.

In this study, we retrospectively compared the time course of clinical and radiological results between knees with $\leq 3^{\circ}$ deviation from the neutral mechanical axis and those with $>3^{\circ}$ deviation after TKAs using the rotating-platform LCS posterior stabilized system. We hypothesized that the LCS system would yield more favorable clinical and radiological results in knees with $\leq 3^{\circ}$ deviation from the neutral mechanical axis in the time course analysis.

\section{Materials and Methods}

This is a retrospective study of 260 consecutive primary TKAs that were performed by a single surgeon using the rotating- platform posterior stabilization LCS system in 215 patients between February 2003 and February 2006. The surgery was bilateral in 45 patients (90 knees). Assessments were performed preoperatively, at 6 weeks, 3 months, 6 months, and 1 year after surgery, and yearly thereafter. Exclusion criteria were bone graft due to rheumatoid arthritis, severe deformity or bone defect, revision surgery, and body mass index (BMI) of over $30 \mathrm{~kg} / \mathrm{m}^{2}$. At the time of evaluation, 8 patients (14 knees) died of reasons not related to surgery, 17 patients ( 22 knees) did not return for follow-up assessments and 18 patients (18 knees) were diagnosed with rheumatoid arthritis. Finally, 172 patients (203 knees) were followed for more than 5 years and were evaluated clinically. To evaluate our hypothesis, the patients were classified according to the degree of deviation from the neutral mechanical axis on the immediate postoperative standing full-length radiograph as Group 1 (patients with $\leq 3^{\circ}$ deviation) or Group 2 (patients with $>3^{\circ}$ deviation). Patient demographics are listed in Table 1 . The primary outcome measures were clinical and radiological results according to the degree of deviation (either $\leq 3^{\circ}$ or $>3^{\circ}$ ) from the neutral mechanical axis.

The operations were performed using a modified subvastus approach through a midline skin incision measuring about $10 \mathrm{~cm}$ in length ${ }^{15}$. The modified subvastus approach features a $2-3 \mathrm{~cm}$ additional snip at the tendinous junction of the vastus medialis obliquus. The cruciate ligaments were excised in all patients. Tibial cutting was initially performed using the extramedullary method followed by a femoral anterior and posterior cut using a gap technique with a lamina spreader or gap blocks. Soft tissue balancing was performed using a sequence of tissue releases for medial, lateral, and posterior structures. Adequate soft tissue balancing was achieved and confirmed by palpation of ligamentous tension and assessment of the gap during symmetrical leg dis-

Table 1. Patient Demographics

\begin{tabular}{|c|c|c|c|c|c|c|}
\hline Variable & Original cohort & Final follow-up cohort & p-value & Group $1^{\text {a) }}$ & Group $2^{\text {b) }}$ & $\mathrm{p}$-value \\
\hline No. of patients & 215 & 172 & & 124 & 48 & \\
\hline No. of knees & 260 & 203 & & 145 & 58 & \\
\hline Age (yr) & $64.8(3.5)$ & $65.9(3.4)$ & NS & $65.1(3.5)$ & $65.4(3.7)$ & NS \\
\hline $\mathrm{M}: \mathrm{F}$ & $7: 208$ & $6: 166$ & NS & $4: 120$ & $2: 46$ & NS \\
\hline Body mass index $\left(\mathrm{kg} / \mathrm{m}^{2}\right)$ & $25.3(1.2)$ & $25.7(1.0)$ & NS & $25.3(1.3)$ & $25.1(1.1)$ & NS \\
\hline Preop mechanical axis $\left({ }^{\circ}\right)$ & & $11.8(4.9)$ & & $11.4(3.4)$ & $12.1(5.4)$ & NS \\
\hline Preop range of motion $\left({ }^{\circ}\right)$ & & $112.7(7.8)$ & & $113.8(6.5)$ & $112.1(9.3)$ & NS \\
\hline
\end{tabular}

Values are presented as mean (standard deviation).

NS: not significant, Preop: preoperative.

${ }^{a}$ Patients with $\leq 3^{\circ}$ deviation of coronal alignment at final follow-up.

${ }^{b)}$ Patients with $>3^{\circ}$ deviation of coronal alignment at final follow-up. 
traction using the laminar spreader. During gap balancing, mediolateral and flexion-extension gaps were adjusted to be within $3 \mathrm{~mm}$ by the laminar spreader. None of the patients underwent patella resurfacing. All components were fixed with cement.

The postoperative protocol for both groups was identical. Straight leg raising exercises were encouraged immediately after surgery. All patients began partial weight-bearing with walking aids and range of motion (ROM) exercises on the second day after surgery. The mechanical axis was measured as the angle between a line drawn from the center of the femoral head to the deepest point of the femoral notch and a line drawn from the midpoint of the tibiotalar joint to the center of the tibial plateau on the standing full-length radiograph.

Pre- and postoperative scores were obtained at every follow-up from all patients using the Knee Society score (KSS) and Hospital for Special Surgery (HSS) systems, and Western Ontario and McMaster Universities (WOMAC) scores were obtained before surgery and 5 years after surgery. Serial postoperative radiographs were evaluated according to the KSS system for radiolucency at the bone-cement interface, wear of polyethylene, any change in the component position, alignment, and osteolysis.

\section{Statistical Analysis}

Survival analysis was performed using Kaplan and Meier ${ }^{16}$. The survival end point was defined as revision surgery for any reason or a recommendation for revision surgery for a mechanical reason. The primary outcome measures were the KSS and HSS score at the final follow-up. Allocation ratio was set at 1:1 and sample size calculation was based on pilot measurements in 30 patients per each group. The standard deviation was 3.13 in Group 1 and 4.83 in Group 2, and the minimum detectable difference for power analysis was calculated to be 5 points in clinical scores. A two-sided test with an $\alpha$ error of $5 \%$ and a $\beta$ error of $20 \%$ were used to determine significant difference. Based on these calculations, the required study size was 57 per each group. The data were analyzed by fitting separate linear mixed models for evaluating time dependent change of the KSS and HSS scores. Posthoc comparisons between the main effects of all pairs of points in time including age, gender, BMI, and preoperative mechanical axis and clinical scores were performed separately for each model, corresponding to the KSS and HSS scores. Bonferroni adjustments were applied to the p-values to account for multiple testing. The significance of $\mathrm{p}$-value was set at 0.05 . The differences according to age, gender, and disease were analyzed using paired t-tests, Student's t-tests, and Mann-Whitney tests, and chisquare tests according to the data form. To improve the test-retest intraobserver reliability, measurements were performed twice by two orthopedic surgeons each with an interval of 2 weeks. The intra- and interobserver reliability was calculated using the intraclass correlation coefficient (ICC) for consistency. Statistical analyses were performed using SPSS ver. 17.0 (SPSS Inc., Chicago, IL, USA).

\section{Results}

\section{Survival Rate and Complications}

The overall prosthesis survival rate was $97.1 \%$ at 6 years with the endpoint defined as revision ( 6 of 203 knees) required for failure of any component due to bearing dislocation, fracture, or deep infection. In Group 1, the estimated survival rate was 97.2\% with a revision rate of $2.8 \%$ ( 4 of 145 knees). In Group 2, the estimated survival rate was $96.6 \%$ with a revision rate of $3.4 \%$ (2 of 58 knees), indicating no statistically significant intergroup difference ( $\mathrm{p}=0.803)$ (Fig. 1$)$.

Postoperative chronic infections were found in four knees over 6 months after TKA (three in Group 1 and one in Group 2). In Group 1, one complication was a postoperative periprosthetic fracture with extension to prosthesis. In Group 2, one complication was a tibial bearing dislocation (spin-out) with polyethylene wear. There were no patellar problems such as fracture, dislocation, and mal-tracking.

\section{Clinical Results}

The linear mixed models analysis revealed that there were significant differences in the KSS and HSS scores during the seven measurement points (before surgery and 6 weeks, 3 months, 6

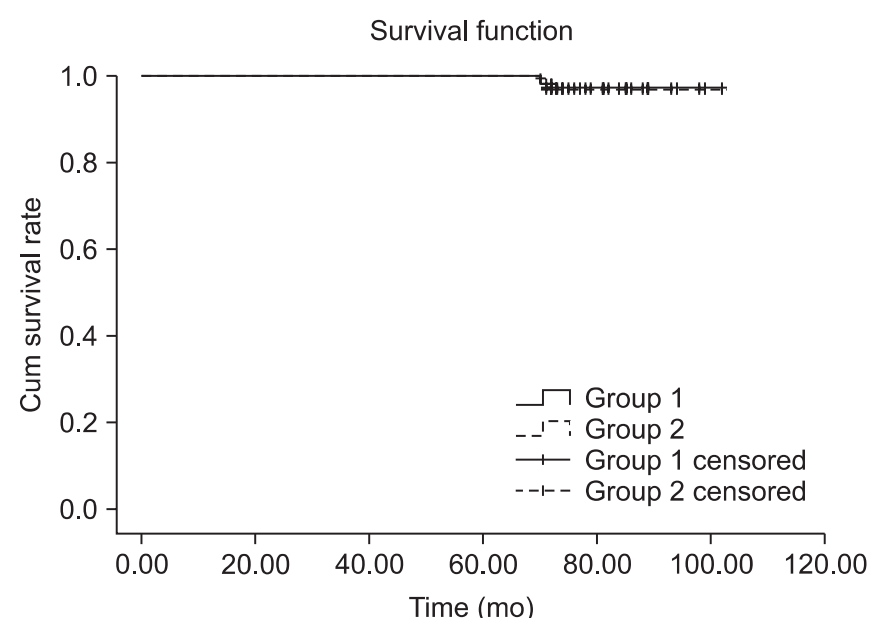

Fig. 1. Kaplan and Meier ${ }^{16)}$ survival analysis. 


\section{Lee et al. Time Dependent Improvement in TKA with LCS System}

months, 1 year, 3 years, and over 5 years after surgery) in both groups $(\mathrm{p}<0.001)$.

In Group 1, the mean KSS continued to improve at all time points until 5 years after surgery, but minimal changes were seen 6 months after surgery. Pairwise comparisons revealed statistically significant difference until 6 months after surgery $(\mathrm{p}<0.001)$, but not thereafter. Using the highest scoring values as a reference, the percentages of improvement in KSS were $59 \%$ at 6 weeks, $68 \%$ at 3 months, $90 \%$ at 6 months, $91 \%$ at 1 year, $95 \%$ at 3 years, and $100 \%$ at over 5 years after surgery (Fig. 2).

The mean HSS functional score in Group 1 continued to im-

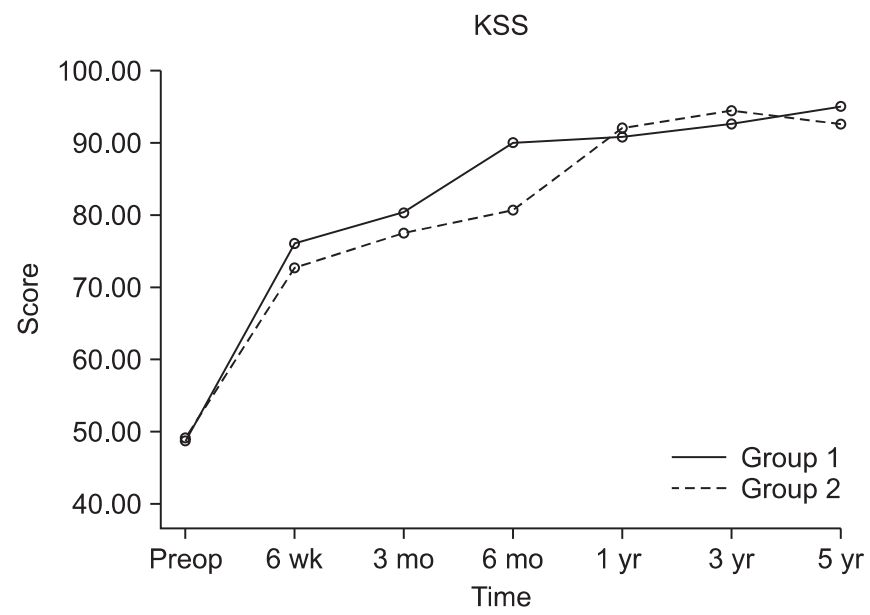

Fig. 2. The graph shows improvement in the Knee Society score (KSS) with time in both groups. In Group 1, pairwise comparisons revealed statistically significant improvement between each time point from preoperative (preop) period to 6 months after surgery $(\mathrm{p}<0.001)$. In Group 2, pairwise comparisons revealed statistically significant improvement between each time point from preop period to 1 year after surgery $(\mathrm{p}<0.001)$. The KSS decreased slightly between the 3- to 5-year followup in Group 2 although no statistical significance was found. prove until 3 years after surgery, but minimal changes were seen from 6 months after surgery. Pairwise comparisons revealed statistically significant difference until 6 months after surgery $(\mathrm{p}<0.001)$, but not since 6 months after surgery. Using the highest scoring values as a reference, the percentages of improvement in HSS knee score were $51 \%$ at 6 weeks, $62 \%$ at 3 months, $87 \%$ at 6 months, $93 \%$ at 1 year, $100 \%$ at 3 years, and $95 \%$ at over 5 years after surgery (Fig. 3).

In Group 2, the mean KSS continued to improve until 3 years after surgery, and then declined until over 5 years after surgery. Only small changes were observed 1 year after surgery. Pairwise

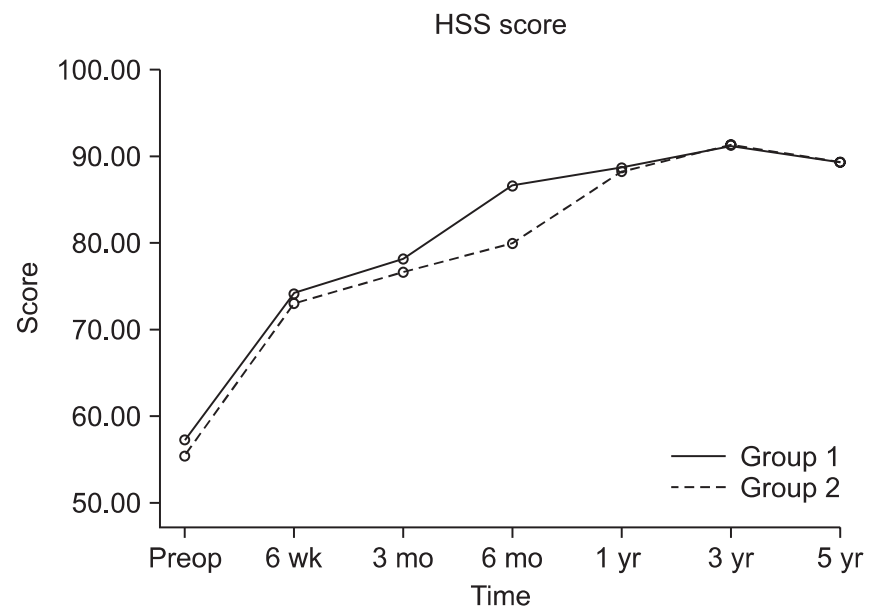

Fig. 3. The graph shows improvement in the Hospital for Special Surgery (HSS) score with time. In Group 1, pairwise comparisons revealed statistically significant improvement between each time point from preoperative (preop) period to 6 months after surgery $(\mathrm{p}<0.001)$. In Group 2, pairwise comparisons revealed statistically significant improvement between each time point from preop period to 1 year after surgery $(\mathrm{p}<0.001)$. The HSS scores slightly decreased in both groups between 3to 5-year follow-up although no significant difference was found.

Table 2. Clinical Outcomes at Each Follow-up Period

\begin{tabular}{|c|c|c|c|c|c|c|}
\hline \multirow{2}{*}{ Variable } & \multicolumn{3}{|c|}{ Knee Society score } & \multicolumn{3}{|c|}{ Hospital for Special Surgery score } \\
\hline & Group 1 & Group 2 & p-value $e^{a)}$ & Group 1 & Group 2 & p-value $e^{a)}$ \\
\hline Preoperative & $48.5 \pm 5.43$ & $49.1 \pm 5.12$ & NS & $55.1 \pm 5.51$ & $54 \pm 5.21$ & NS \\
\hline $6 \mathrm{wk}$ & $76.3 \pm 13.1$ & $72.4 \pm 10.2$ & NS & $74.8 \pm 8.12$ & $73.5 \pm 9.42$ & NS \\
\hline $3 \mathrm{mo}$ & $81.3 \pm 7.94$ & $77.7 \pm 6.25$ & $<0.001$ & $77.9 \pm 7.54$ & $77.1 \pm 8.19$ & NS \\
\hline $6 \mathrm{mo}$ & $90.9 \pm 5.36$ & $81.9 \pm 6.71$ & $<0.001$ & $87.1 \pm 8.61$ & $79.1 \pm 8.33$ & $<0.001$ \\
\hline $1 \mathrm{yr}$ & $91.5 \pm 6.18$ & $92.6 \pm 8.38$ & NS & $88.5 \pm 9.31$ & $88.3 \pm 8.71$ & NS \\
\hline $3 \mathrm{yr}$ & $93.1 \pm 9.81$ & $94.1 \pm 9.48$ & NS & $90.7 \pm 13.22$ & $90.2 \pm 13.35$ & NS \\
\hline Over 5 yr & $95.1 \pm 9.23$ & $92.9 \pm 9.52$ & NS & $89.8 \pm 10.2$ & $89.5 \pm 11.1$ & NS \\
\hline
\end{tabular}

Values are presented as mean \pm standard deviation.

NS: not significant.

${ }^{a)} \mathrm{p}$-value for comparing two groups at each period. 
Table 3. Preoperative (preop) and Postoperative (postop) WOMAC Scores

\begin{tabular}{lccc}
\hline \multicolumn{1}{c}{ Variable } & Group 1 & Group 2 & p-value \\
\hline Preop WOMAC scores & & & \\
Total & $56.2 \pm 17.5$ & $61.0 \pm 15.5$ & NS \\
Pain & $9.4 \pm 3.5$ & $10.0 \pm 3.9$ & NS \\
Stiffness & $5.0 \pm 1.4$ & $4.8 \pm 1.4$ & NS \\
Function & $41.7 \pm 16.6$ & $46.2 \pm 13.1$ & NS \\
Postop WOMAC scores & & & \\
Total & $11.3 \pm 5.6$ & $11.5 \pm 7.3$ & NS \\
Pain & $2.7 \pm 3.1$ & $3.1 \pm 2.5$ & NS \\
Stiffness & $1.5 \pm 1.1$ & $1.7 \pm 1.2$ & NS \\
Function & $9.1 \pm 8.5$ & $9.7 \pm 10.1$ & NS \\
Postop ROM $\left({ }^{\circ}\right)$ & $126.5 \pm 6.8$ & $125.1 \pm 7.1$ & NS \\
\hline
\end{tabular}

Values are presented as mean \pm standard deviation.

WOMAC: Western Ontario and McMaster Osteoarthritis, NS: not significant, ROM: range of motion.

comparisons revealed statistically significant difference until 1 year after surgery $(p<0.001)$, but not thereafter. Using the highest scoring values as a reference, the percentages of improvement in KSS were $52 \%$ at 6 weeks, $62 \%$ at 3 months, $70 \%$ at 6 months, $96 \%$ at 1 year, $100 \%$ at 3 years, and $96 \%$ at over 5 years after surgery (Fig. 2).

The mean HSS functional score in Group 2 continued to improve until 3 years after surgery, and then declined until 5 years after surgery. Only small changes were observed 1 year after surgery. Pairwise comparisons revealed statistically significant difference until 1 year after surgery $(\mathrm{p}<0.001)$, but not since 1 year after surgery. Using the highest scoring values as a reference, the percentages of improvement in HSS knee score were $49 \%$ at 6 weeks, $59 \%$ at 3 months, $68 \%$ at 6 months, $91 \%$ at 1 year, $100 \%$ at 3 years, and $94 \%$ at over 5 years after surgery (Fig. 3 ).

Comparison of the two groups showed no statistically significant difference in the preoperative KSS and HSS scores (both, $\mathrm{p}>0.05$ ) and postoperative scores (both, $\mathrm{p}>0.05$ ) since 1-year after surgery. However, there were significant intergroup differences between the 6 weeks postoperative 1 year postoperative period ( $\mathrm{p}<0.001$ ) (Table 2 ). The preoperative and final follow-up WOMAC scores and ROM were not significantly different between groups (Table 3).

\section{Radiological Evaluation}

The mean preoperative mechanical femorotibial angle was $11.8^{\circ} \pm 4.85^{\circ}$ (mean \pm standard deviation) varus, whereas the me-
Table 4. Comparison of Radiolucent Lines between Groups

\begin{tabular}{lcccc}
\hline \multirow{2}{*}{ Variable } & \multicolumn{3}{c}{ Location of radiolucency } & \\
\cline { 2 - 4 } & Femur & Tibia & Femur and Tibia & \\
\hline Group 1 & 0 & 3 & 0 & $2.0(3 / 145)$ \\
Group 2 & 1 & 6 & 2 & $15.5(9 / 58)$ \\
\hline
\end{tabular}

dian final follow-up mechanical femorotibial angle was $2.1^{\circ} \pm 5.74^{\circ}$ varus. The mean preoperative mechanical femorotibial angle was $11.4^{\circ} \pm 3.43^{\circ}$ varus in Group 1 and $12.1^{\circ} \pm 5.41^{\circ}$ varus in Group 2 ( $p>0.05$ ). The mean postoperative mechanical femorotibial angle was $1.5^{\circ} \pm 1.1^{\circ}$ varus in Group 1 and $4.8^{\circ} \pm 2.12^{\circ}$ varus in Group $2(\mathrm{p}=0.001)$. Radiolucent lines were observed in 3 of 145 knees (2.0\%) in Group 1 and in 9 of 58 knees (15.5\%) in Group 2 on the final follow-up radiographs. The incidence of radiolucency was higher in Group 2 than in Group 1 ( $\mathrm{p}=0.001$ ) (Table 4). Regarding zonal distribution, radiolucent lines were observed in zones I, II, and IV on the femoral side in Group 2 and in zones I or II on the tibial side in both groups. However, no clinically significant progressive radiolucent lines were observed and all radiolucent lines were less than $2 \mathrm{~mm}$.

The ICC for inter- and intra-observer reliability ranged from 0.72 to 0.88 for all measurements, indicating that all measurements had good inter-observer reliability.

\section{Discussion}

A biomechanical study of mobile-bearing LCS TKA has revealed that a congruency and mobility of femorotibial articulation with a rotating-platform can reduce contact stresses within the polyethylene liner and allow axial rotation ${ }^{17)}$. This leads to reduced torque forces on the tibial component, eventually resulting in good long-term survivorship ${ }^{2-5)}$. Other mobile-bearing designs have also been confirmed to lower wear rates and the risk of associated osteolysis in radiological studies ${ }^{18,19)}$.

The results of the current study are consistent with those of the previous studies ${ }^{3-5)}$ : the use of the LCS system in TKA resulted in acceptable clinical and radiographic results for more than 5 years of follow-up regardless of the degree of coronal mechanical axis. Overall, radiolucent lines were observed in $5.9 \%$ of the total patients, although the incidence of radiolucency was higher in the group with $\geq 3^{\circ}$ deviation from the neutral mechanical axis (15.5\%) than in the group with $<3^{\circ}$ deviation. However, there was significant difference between the two groups in the improvement of clinical scores during the first year after TKA. The overall survival rate was $97.1 \%$ at 6 years without any true loosening. 


\section{Lee et al. Time Dependent Improvement in TKA with LCS System}

Proper alignment in TKA has been the subject of debate. Incorrect alignment can lead to abnormal prosthesis wear, premature mechanical loosening of the components, and patellofemoral problems ${ }^{7,8)}$. Thus, most surgeons have tried to achieve a neutral mechanical axis. A recent study supported this concept: accurate coronal alignment of the total knee prosthesis (within $3^{\circ}$ of neutral mechanical axis) resulted in greater functional improvement and more favorable quality of life up to 5 years postoperatively ${ }^{20}$. Yet, other studies proposed the importance of other factors that may influence TKA outcomes and suggested that improved component design and surgical technique reduced the risk of negative clinical outcomes associated with poor coronal alignment ${ }^{14,21}$. However, neutral coronal alignment does not always guarantee the accurate position or rotation of each component. Bonner et $\mathrm{al}^{22)}$ investigated the relationship between the survival of primary TKA using a cruciate-retaining press-fit condylar system (Johnson and Johnson Professional Inc., Raynham, MA, USA) and mechanical axis alignment: the relationship was weaker than that described in a number of previous reports ${ }^{22)}$. Their results are in agreement with our study results except for the time course of the clinical improvement and fixed- bearing TKA. In our study, postoperative coronal alignment exceeded $3^{\circ}$ of varus/valgus deviation in $28 \%$ of the patients operated by the conventional gap technique, as in previous studies where the percentages were $25 \%-26 \%{ }^{23,24)}$.

The more than 5-year follow-up clinical results of this study demonstrated that favorable results could be obtained with more than $3^{\circ}$ of varus/valgus deviation of coronal alignment. These results were consistent with those of the study by Vogt and Saarbach $^{24)}$. Moreover, there was substantial improvement in the KSS and HSS scores as early as 6 weeks postoperatively: approximately $50 \%$ improvement in the KSS and HSS scores was achieved by 6 weeks in both groups, and the scores gradually improved during the follow-up. As in previous studies ${ }^{11,12,25)}$, most expected recovery was obtained within first year on account of improvement of ROM, recovery of quadriceps power, and reduced pain and swelling $^{10-12,25)}$. However, in our study, the time of improvement was much faster in Group 1 than Group 2, despite the similarity of the final outcomes. Moreover, clinical KSS and HSS scores slightly declined after 3 years in Group 2, even though the decrease was not statistically significant. These similar clinical results of TKA regardless of the coronal alignment deviation can be explained by the concept of constitutional varus alignment ${ }^{13)}$. The degree of varus deformity is different in each individual; thus, the proper postoperative alignment of the mechanical axis is different from person to person. In our study, comparable clinical scores were obtained in patients with the mechanical axis deviation greater than the traditional criteria, if proper soft tissue balancing was achived.

From the radiological point of view, the incidence of radiolucency was higher in Group 2 than Group 1 at the final follow-up. Many studies have associated low rates of osteolysis and loosening with the LCS system ${ }^{3,26)}$ except for one study where the rate was $2 \%{ }^{27)}$. In the current study, although no clinical loosening or osteolysis was evident in either group, the incidence of radiolucency was much higher in Group 2. Moreover, most radiolucent lines were observed on the tibial side as in previous studies ${ }^{18,24)}$, although it was impossible to identify whether these lines could affect clinical outcomes or further survival rates of TKA ${ }^{18,28)}$. Recently, Sadoghi et al. ${ }^{29)}$ reported that radiolucent lines in the tibial component could represent poor implant seating, not loosening or osteolysis, and could be associated with knee pain. According to the concept of constitutional varus alignment ${ }^{13)}$, the appropriate criteria for mechanical axis could be different for each individual. However, in terms of the weight-bearing and load distribution mechanism, the traditional criteria for mechanical axis would have the biomechanical advantages including decreased varus momentum ${ }^{30)}$ and result in lower rates of radiolucency that have been associated with the risk of loosening. Our study results demonstrated that constitutional varus knees can achieve comparable clinical outcomes with proper soft tissue balancing, but not mechanical advantages. However, our study did not reveal any relationship between radiolucency and clinical scores or survival outcomes. A longer term follow-up would be necessary to evaluate the relationship between loosening, clinical outcomes and changes in radiolucent lines or osteolysis.

This study had some limitations. First, this study is retrospective. However, the data was collected prospectively, the cases that have missing data for follow-up were excluded, and this study has shown enough power of study. The second limitation is the lack of standardization of the force applied by the spreader in the balancing procedure that may have affected the final outcome. However, all TKAs were performed by one senior surgeon, and all posterior cruciate ligaments were sacrificed with sequential soft tissue release to control the bias. Third, the duration of followup was 5 years, so the long-term variability in the outcome could not be assessed. Although there were no statistically significant intergroup differences in the clinical outcomes and survival rates regardless of the coronal alignment during the 5-year follow-up, some differences may occur due to the coronal alignment in the long term. Fourth, although $3^{\circ}$ deviation of coronal alignment was used as a cut off value, there could be another value that may 
reflect the influence of the coronal alignment deviation on the clinical outcome. However, increasing the cut off value would decrease the number of cases, resulting in low power of study. Moreover, the $3^{\circ}$ deviation of coronal alignment has been used as the criteria for TKA at our institution.

One of the strengths of our study is that we included both short-term and intermediate-term evaluations to establish a true timeline for functional recovery. Moreover, the survival rate and radiolucency were analyzed in the intermediate-term follow-up.

\section{Conclusions}

Functional results and recovery after TKA using LCS are timedependent. Substantial improvements in KSS and HSS scores were present as early as 6 weeks after surgery, and most of the expected improvements were achieved by 6 months after surgery in patients with $\leq 3^{\circ}$ deviation of coronal alignment and by 1 year after surgery in patients with $>3^{\circ}$ deviation. The findings of the present intermediate-term, retrospective cohort study suggest that the rotating-platform LCS posterior stabilization system performs well regardless of the degree of coronal alignment deviation without causing significant differences in the clinical outcome and survival rate over 5 years of follow-up.

\section{Conflict of Interest}

No potential conflict of interest relevant to this article was reported.

\section{References}

1. Epinette JA, Manley MT. Hydroxyapatite-coated total knee replacement: clinical experience at 10 to 15 years. J Bone Joint Surg Br. 2007;89:34-8.

2. Baker PN, Khaw FM, Kirk LM, Esler CN, Gregg PJ. A randomised controlled trial of cemented versus cementless press-fit condylar total knee replacement: 15-year survival analysis. J Bone Joint Surg Br. 2007;89:1608-14.

3. Buechel FF Sr, Buechel FF Jr, Pappas MJ, D’Alessio J. Twentyyear evaluation of meniscal bearing and rotating platform knee replacements. Clin Orthop Relat Res. 2001;(388):41-50.

4. Callaghan JJ, Squire MW, Goetz DD, Sullivan PM, Johnston RC. Cemented rotating-platform total knee replacement: a nine to twelve-year follow-up study. J Bone Joint Surg Am. 2000;82:705-11.

5. Huang $\mathrm{CH}$, Ma HM, Lee YM, Ho FY. Long-term results of low contact stress mobile-bearing total knee replacements. Clin Orthop Relat Res. 2003;(416):265-70.

6. Insall J, Scott WN, Ranawat CS. The total condylar knee prosthesis: a report of two hundred and twenty cases. J Bone Joint Surg Am. 1979;61:173-80.

7. Werner FW, Ayers DC, Maletsky LP, Rullkoetter PJ. The effect of valgus/varus malalignment on load distribution in total knee replacements. J Biomech. 2005;38:349-55.

8. Sikorski JM. Alignment in total knee replacement. J Bone Joint Surg Br. 2008;90:1121-7.

9. Bathis H, Perlick L, Tingart M, Luring C, Zurakowski D, Grifka J. Alignment in total knee arthroplasty. A comparison of computer-assisted surgery with the conventional technique. J Bone Joint Surg Br. 2004;86:682-7.

10. KAT Trial Group, Johnston L, MacLennan G, McCormack K, Ramsay C, Walker A. The Knee Arthroplasty Trial (KAT) design features, baseline characteristics, and two-year functional outcomes after alternative approaches to knee replacement. J Bone Joint Surg Am. 2009;91:134-41.

11. Kennedy DM, Hanna SE, Stratford PW, Wessel J, Gollish JD. Preoperative function and gender predict pattern of functional recovery after hip and knee arthroplasty. J Arthroplasty. 2006;21:559-66.

12. Kennedy DM, Stratford PW, Riddle DL, Hanna SE, Gollish JD. Assessing recovery and establishing prognosis following total knee arthroplasty. Phys Ther. 2008;88:22-32.

13. Bellemans J, Colyn W, Vandenneucker H, Victor J. The Chitranjan Ranawat award: is neutral mechanical alignment normal for all patients? The concept of constitutional varus. Clin Orthop Relat Res. 2012;470:45-53.

14. Parratte S, Pagnano MW, Trousdale RT, Berry DJ. Effect of postoperative mechanical axis alignment on the fifteen-year survival of modern, cemented total knee replacements. J Bone Joint Surg Am. 2010;92:2143-9.

15. Jung YB, Lee YS, Lee EY, Jung HJ, Nam CH. Comparison of the modified subvastus and medial parapatellar approaches in total knee arthroplasty. Int Orthop. 2009;33:419-23.

16. Kaplan EL, Meier P. Nonparametric estimation from incomplete observations. J Am Stat Assoc. 1958;53:457-81.

17. Pappas MJ. Engineering design of the LCS knee replacement. In: Hamelynck KJ, Stiehl JB, eds. LCS mobile bearing knee arthroplasty: a 25 years worldwide review. Berlin: Springer; 2002. p39-52.

18. Kim YH, Kim JS. Comparison of anterior-posterior-glide and rotating-platform low contact stress mobile-bearing total knee arthroplasties. J Bone Joint Surg Am. 2004;86:1239- 
47.

19. Ho FY, Ma HM, Liau JJ, Yeh CR, Huang CH. Mobile-bearing knees reduce rotational asymmetric wear. Clin Orthop Relat Res. 2007;462:143-9.

20. Huang NF, Dowsey MM, Ee E, Stoney JD, Babazadeh S, Choong PF. Coronal alignment correlates with outcome after total knee arthroplasty: five-year follow-up of a randomized controlled trial. J Arthroplasty. 2012;27:1737-41.

21. Astephen Wilson JL, Wilson DA, Dunbar MJ, Deluzio KJ. Preoperative gait patterns and BMI are associated with tibial component migration. Acta Orthop. 2010;81:478-86.

22. Bonner TJ, Eardley WG, Patterson P, Gregg PJ. The effect of post-operative mechanical axis alignment on the survival of primary total knee replacements after a follow-up of 15 years. J Bone Joint Surg Br. 2011;93:1217-22.

23. Petersen TL, Engh GA. Radiographic assessment of knee alignment after total knee arthroplasty. J Arthroplasty. 1988; 3:67-72.

24. Vogt JC, Saarbach C. LCS mobile-bearing total knee replacement. A 10-year's follow-up study. Orthop Traumatol Surg Res. 2009;95:177-82.

25. Nilsdotter AK, Toksvig-Larsen S, Roos EM. A 5 year pro- spective study of patient-relevant outcomes after total knee replacement. Osteoarthritis Cartilage. 2009;17:601-6.

26. Jordan LR, Olivo JL, Voorhorst PE. Survivorship analysis of cementless meniscal bearing total knee arthroplasty. Clin Orthop Relat Res. 1997;(338):119-23.

27. Sanchez-Sotelo J, Ordonez JM, Prats SB. Results and complications of the low contact stress knee prosthesis. J Arthroplasty. 1999;14:815-21.

28. Hooper G, Rothwell A, Frampton C. The low contact stress mobile-bearing total knee replacement: a prospective study with a minimum follow-up of ten years. J Bone Joint Surg Br. 2009;91:58-63.

29. Sadoghi P, Leithner A, Weber P, Friesenbichler J, Gruber G, Kastner N, Pohlmann K, Jansson V, Wegener B. Radiolucent lines in low-contact-stress mobile-bearing total knee arthroplasty: a blinded and matched case control study. BMC Musculoskelet Disord. 2011;12:142.

30. Hilding MB, Lanshammar H, Ryd L. Knee joint loading and tibial component loosening. RSA and gait analysis in 45 osteoarthritic patients before and after TKA. J Bone Joint Surg Br. 1996;78:66-73. 